\title{
Reporting elevated vancomycin minimum inhibitory concentration in methicillin-resistant Staphylococcus aureus: consensus by an International Working Group
}

\author{
Mark Wilcox', Suleiman Al-Obeid², Ana Gales³, Roman Kozlov4, José A Martínez-Orozco ${ }^{5}$, \\ Flavia Rossi ${ }^{6}$, Sergey Sidorenko ${ }^{7}$ \& Joseph Blondeau*,8 \\ ${ }^{1}$ Leeds Teaching Hospitals NHS Trust \& University of Leeds, Leeds, UK \\ ${ }^{2}$ Microbiology Department, Security Forces Hospital, Riyadh, Saudi Arabia \\ ${ }^{3}$ Division of Infectious Diseases, Escola Paulista de Medicina, Universidade Federal de São Paulo, São Paulo, Brazil \\ ${ }^{4}$ Institute of Antimicrobial Chemotherapy, Smolensk State Medical University, Smolensk, Russia \\ ${ }^{5}$ Infectious Diseases \& Clinical Microbiology Department, National Institute of Respiratory Diseases, Mexico City, Mexico \\ ${ }^{6}$ Hospital das Clínicas da Faculdade de Medicina, Seção de Microbiologia, Divisão de Laboratório Central LIM03, Universidade de \\ São Paulo, São Paulo, Brazil \\ ${ }^{7}$ Department of Medical Microbiology \& Molecular Epidemiology, Pediatric Research \& Clinical Center for Infectious Diseases, \\ Department of Medical Microbiology, North-Western State Medical University named after I.I. Mechnikov, Saint Petersburg, Russia \\ ${ }^{8}$ Department of Clinical Microbiology, Royal University Hospital, Saskatchewan, Canada \\ *Author for correspondence: Tel.: +1 306655 6943; Fax: +1 306655 6947; Joseph.Blondeau@saskhealthauthority.ca
}

\begin{abstract}
Methicillin-resistant Staphylococcus aureus (MRSA) remains an important cause of serious infection, for which vancomycin is often recommended as the first-choice antibiotic treatment. Appropriate vancomycin prescribing requires accurate measurement of minimum inhibitory concentrations (MICs) to avoid treatment failure, and yet determination can be challenging due to methodological difficulties associated with susceptibility testing. An International Working Group of infectious disease specialists and clinical/medical microbiologists reached a consensus that empirical MRSA infection therapies should be chosen regardless of the suspected origin of the infecting strain (e.g., community or hospital) due to the complex intermingling epidemiology of MRSA clones in these settings. Also, if an elevated vancomycin MIC in the susceptible range is obtained in routine testing, an alternative second method should be used for confirmation and to aid antibiotic therapy recommendations. There is no absolutely dependable method for the accurate determination of vancomycin MIC, but broth microdilution appears to be the most reliable.
\end{abstract}

First draft submitted: 14 December 2018; Accepted for publication: 21 January 2019; Published online: 6 February 2019

Keywords: CA-MRSA • HA-MRSA • minimum inhibitory concentration $\bullet$ susceptibility $\bullet$ vancomycin

Methicillin-resistant Staphylococcus aureus (MRSA) remains one of the most important causes of serious infection, particularly among hospitalized patients. MRSA infections are also associated with poorer outcomes than those caused by methicillin-sensitive $S$. aureus infections [1,2]. Therefore, understanding and immediately recognizing risk factors for hospital-acquired/healthcare-associated (HA) MRSA [3] are considered vital to improve management of the patients at risk of MRSA infection until culture confirmation [4]. Nevertheless, the epidemiology of MRSA has changed over the past decade, with infections also now being observed in community settings [5]. However, the value of differentiating between the origin/place of onset of the infection for disease diagnosis and treatment management is uncertain.

Guidelines recommend the use of vancomycin, linezolid, daptomycin, telavancin and clindamycin to treat serious MRSA infections, with minocycline, doxycycline and clindamycin used for less severe infections [6]. In recent years, several new antibiotics with high potency against MRSA have been approved for the treatment of acute bacterial skin and skin structure infections, including ceftaroline, dalbavancin, tedizolid, oritavancin and delafloxacin [7-9]. While 
these newer agents provide useful alternative therapies for multidrug-resistant pathogens and treatment benefits for specific patient groups, vancomycin still remains the first-choice antibiotic in most countries for suspected or confirmed MRSA infections. Indeed, vancomycin is recommended by guidelines for both HA-MRSA and community-associated (CA) MRSA infection, despite reports of treatment failures for infection caused by some strains with elevated vancomycin minimum inhibitory concentrations (MICs) [6,7,10]. Appropriate prescribing of vancomycin requires measurement and reporting of elevated MICs, yet vancomycin MIC determination can be challenging due to the limitations associated with available susceptibility testing methods in real medical practice. Consequently, a working group of infectious disease specialists and clinical/medical microbiologists from Brazil, Canada, Mexico, Saudi Arabia, Russia and the UK was convened to discuss elevated vancomycin MIC in both CAMRSA and HA-MRSA, the importance of differentiating between strains, and issues surrounding the discordance between various vancomycin susceptibility testing methods. This article summarizes their recommendations on these matters and suggests the most suitable methodology to adopt, which could enable hospitals to monitor local trends and accurately guide antibiotic therapy for the management of MRSA infections.

\section{Differentiation between CA-MRSA \& HA-MRSA}

MRSA isolates were originally restricted to hospital and other healthcare settings; however, over the past few decades, an increasing proportion of younger patients without known HA-MRSA risk factors have acquired MRSA in community settings (i.e., CA-MRSA). CA-MRSA, at least at the outset, had markedly different antimicrobial susceptibility patterns compared with conventional HA-MRSA [5,11]. Differentiation between CA-MRSA and HA-MRSA infections was considered relevant initially since it was recognized that the former were susceptible to more antibiotic classes than HA-MRSA; consequently, there were more choices of empiric antibiotic therapy for CA-MRSA infections [5]. However, CA-MRSA strains are now acquiring resistance to additional antibiotics, blurring the distinction between the two types [12]. Furthermore, investigations of the genetic background of CA-MRSA, from an epidemiological point of view, also highlight a tendency toward a broader mixing among MRSA clones, with infections acquired in the community setting now being categorized as community-onset (CO) MRSA infections. The distribution of their clonal origin has also shown the emergence of clones that would typically be categorized as HA-MRSA (e.g., CO-HA-MRSA based on SCCmec typing) [5]. Similarly, some studies have demonstrated the emergence of 'typically' CA-MRSA clones in hospitals/healthcare settings (HO) [12-14]. This blurring of the distribution of clones in both settings therefore makes appropriate antibiotic selection more challenging. Mixing of CA-MRSA and HA-MRSA clones has been described globally [12] and contributes to the constant evolution and change of regional and/or local susceptibility patterns [13,14].

While the majority of Working Group members differentiate between CA-MRSA and HA-MRSA, using a variety of methods including clinical findings, time of diagnosis of MRSA infection (e.g., $<48 \mathrm{~h}$ or $>48 \mathrm{~h}$ after hospital admission), antibiogram (local susceptibility pattern) and genetic testing, such differentiation does not play a critical role in antibiotic stewardship programs. Although differentiation between strains remains of scientific interest, particularly from an epidemiological point of view for clinical microbiologists, the group highlight that prescribing physicians generally only require confirmation of the pathogen being an MRSA strain and the antibiotic susceptibility pattern in order to make an informed decision on the most appropriate antibiotic. Furthermore, they stress that reporting to the treating physician of the actual antibiotic susceptibility of culture-confirmed MRSA strains by the microbiologist is more relevant than information on the origin of the clone (e.g., CA-MRSA or HA-MRSA clone).

\section{Reporting susceptibility based on elevated vancomycin MIC}

Vancomycin has been the mainstay antibiotic for the treatment of MRSA infections globally for many years $[6,11,15,16]$, although it has several well-known limitations, including renal toxicity associated with high trough levels, and within the susceptible range it may also be associated with treatment failure and/or resistance emergence during prolonged treatment (e.g., heterogeneous vancomycin-intermediate $S$. aureus) [17-19]. Analyses suggest a correlation between vancomycin MIC and outcome, with a meta-analysis of 22 studies conducted in $S$. aureus bacteremia patients from different locations finding a threefold increase in the risk of treatment failure and increased mortality when MRSA had an elevated vancomycin MIC (i.e., $\geq 1.5 \mu \mathrm{g} / \mathrm{ml}$ ) irrespective of the source of infection or MIC methodology [10]. While the decision to change to an alternative antibiotic should be based on individual circumstances and the clinical response to vancomycin rather than MIC alone, this link between vancomycin MIC and outcome clearly influences antibiotic selection and indeed underlies the recommendation that an 


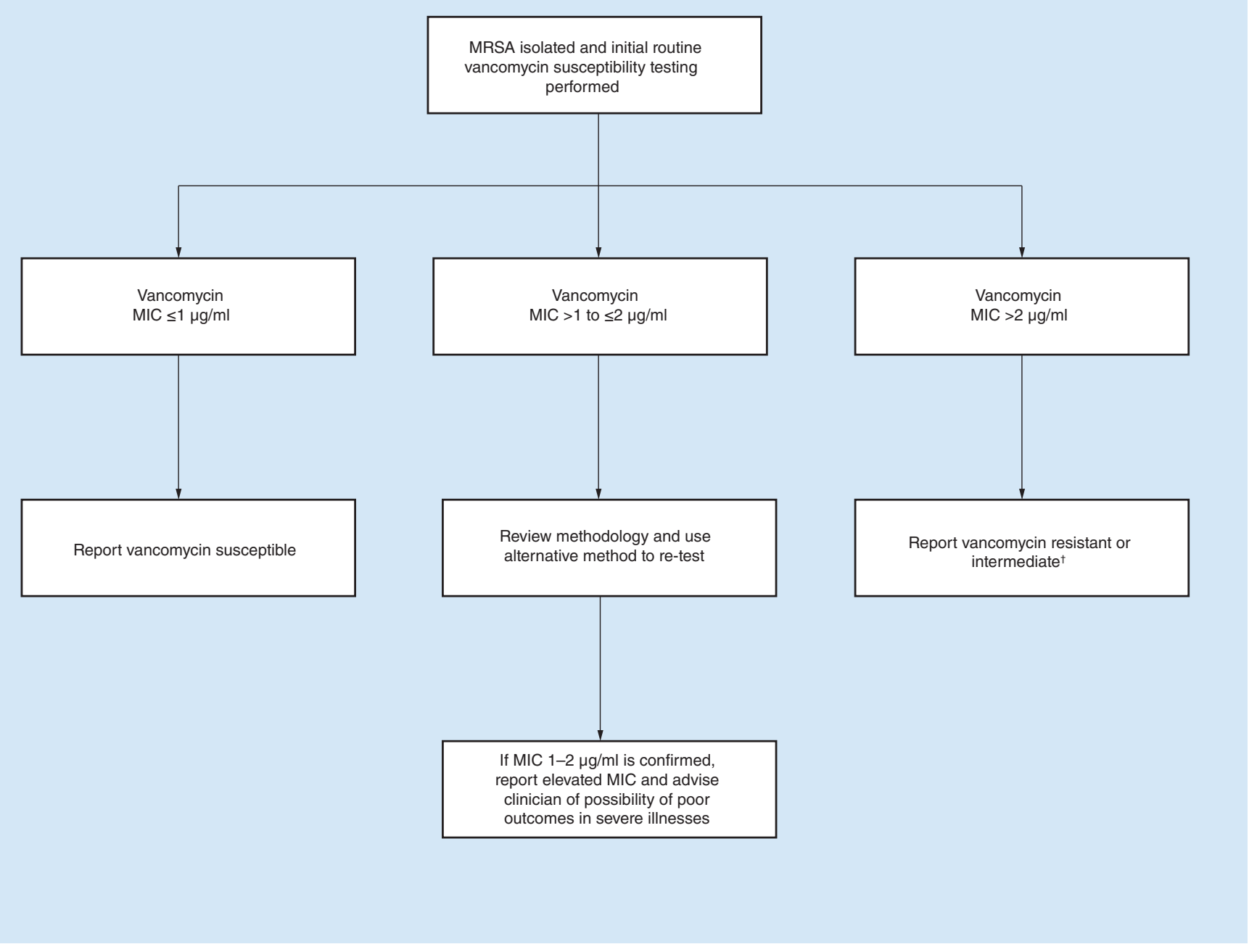

Figure 1. Recommended testing methodology for reporting elevated vancomycin minimum inhibitory concentration in methicillin-resistant Staphylococcus aureus.

${ }^{\dagger}$ EUCAST susceptibility breakpoints: Susceptible: $\leq 2 \mu \mathrm{g} / \mathrm{ml}$, Resistant: $>2 \mu \mathrm{g} / \mathrm{ml}$ [39]; CLSI susceptibility breakpoints: Susceptible: $\leq 2 \mu \mathrm{g} / \mathrm{ml}$, Intermediate: $4-8 \mu \mathrm{g} / \mathrm{ml}$, Resistant: $\geq 16 \mu \mathrm{g} / \mathrm{ml}[37,38]$.

MIC: Minimum inhibitory concentration; MRSA: Methicillin-resistant Staphylococcus aureus.

alternative second-line antibiotic, such as linezolid, tedizolid, daptomycin, telavancin, dalbavancin or clindamycin, to which the infecting isolate is susceptible should be used for isolates with a vancomycin MIC $>2 \mu \mathrm{g} / \mathrm{ml}[6,9,15]$. Indeed, it may be reasonable to consider more potent alternatives for empiric therapy in areas where prevalence of MRSA is high. Nevertheless, accurate measurement of vancomycin MIC is essential in order to ensure correct management of MRSA, reduce inappropriate antibiotic use and lessen the emergence of resistant strains.

The precise determination of vancomycin MIC is complex, due to the limitations of current methods for testing susceptibility [15], some of which may be inherent of the method itself and not linked to the conditions used [20]. A number of methods are currently used for antibiotic susceptibility testing and can be automated (i.e., Vitek ${ }^{\circledR}$ 2, BD Phoenix ${ }^{T M}$, MicroScan, Sensititre ${ }^{T M}$ ) or manual (i.e., disk diffusion, broth microdilution and Etest) [21], although broth microdilution is the standard method used to define vancomycin MIC [15,20]. Importantly, however, discrepancies in MIC values have been reported, with a one to two dilution difference in the obtained MIC values apparent when the results of various methods are compared [20,22,23]. The lack of agreement in vancomycin 
MIC values has been observed in several regions, including Australia [24], India [25], Taiwan [26], the USA [22,27] and Brazil [28]. Based on these investigations, it appears that results from different testing methodologies are consistent for the lower range MICs $(<1 \mu \mathrm{g} / \mathrm{ml})$ although Etest, MicroScan and Phoenix are likely to report higher values (compared with broth microdilution) for MICs $\geq 1 \mu \mathrm{g} / \mathrm{ml}[15,22]$. The broth microdilution method provides the lowest MIC values, but may fail to identify small changes in vancomycin MIC values because the method uses twofold dilutions of vancomycin concentration by convention [15]. Of note, storage may alter the susceptibility readings of MRSA [20,29]. Edwards et al. have found that the Etest method results in higher vancomycin MIC values for freshly isolated MRSA strains compared with those of stored and subsequently tested MRSA strains (within 6 months); this tendency was found to be significant [29]. In contrast, storage does not seem to have a large impact on vancomycin MIC values when measured with broth microdilution up to 6 months but decreased vancomycin MICs were obtained after storing the samples for at least 12 months [20]. Such findings may have implications in retrospective susceptibility measurements or in large surveillance studies with frozen samples being transported to central laboratories.

Lack of consistency in MIC readings obtained by different methodologies may also account for conflicting reports of increases in vancomycin MIC over time in recent years. The existence of MIC creep was reported by some laboratories [30,31], while other studies could not confirm any increase in vancomycin MIC over the years [29,32-34]. Lack of observed creep was also echoed by many Working Group members, who have not found increased incidence of elevated MIC over a 5-year period (data not shown). The discrepancies between published reports of MIC creep may be partly explained by the MIC methodologies used in the different studies, with creep generally observed in smaller single-center studies that employed Etest assays, while negative findings reported by larger multicenter studies may be due to the use of broth microdilution [15]. Such issues also complicate recent reports of elevated vancomycin MICs in both CA-MRSA and HA-MRSA isolates in Russia [35] and Taiwan [36]. In particular, the Taiwanese study, which compared Vitek 2 and Etest with standard broth microdilution methodology in the measurement of vancomycin MIC, found that agreement rates at $2 \mu \mathrm{g} / \mathrm{ml}$ were only $53.6 \%$ for Vitek 2 versus 86.7\% for Etest, with discordance rates being higher in HA isolates than in CA isolates [36]. Thus, the limitations of the susceptibility testing methods in obtaining accurate and reproducible vancomycin MIC for MRSA make comparison between studies implausible to explain the presence or absence of vancomycin MIC creep. This controversial issue may be resolved in a prospective, large multicenter study across various regions and countries which use identical laboratory methods, standardized to a high degree, to measure vancomycin susceptibility on freshly collected MRSA isolates. Furthermore, it is plausible that MIC creep could be associated with some but not all MRSA clones, and so local strain distribution/epidemiology will likely determine whether this phenomenon is measurable and/or observed.

\section{Conclusion \& recommendations}

The Working Group recommends that microbiology laboratories should report the isolation of MRSA and recount the full susceptibility profile of the organism without any differentiation between CA-MRSA and HA-MRSA; it also discourages the use of these terms. The Group further stresses that methodological difficulties associated with susceptibility testing described above create a real challenge for clinicians when selecting the most appropriate antibiotic for the treatment of MRSA infections. While elevated vancomycin MIC poses a risk of treatment failure, the agent remains the standard of care for most MRSA infections, necessitating ongoing monitoring of MIC to ensure effective therapy. Either Clinical Laboratory Standards Institute (CLSI) [37,38] or European Committee on Antimicrobial Susceptibility Testing (EUCAST) [39] breakpoints can be followed for susceptibility testing, although there is no clear guidance currently on the methods to be used to determine MIC. Indeed, the discrepancy among the susceptibility testing methods used at present suggests that the current methodologies may not be optimized to determine vancomycin susceptibility results [29]. Nevertheless, recommendations on the precise method to use to test for vancomycin susceptibility are problematic due to variations in the cost and availability of the various methods in different regions. Consequently, until better susceptibility testing methods are available, hospitals must use the methods currently available to them.

The Group recommends that for vancomycin $\mathrm{MIC} \leq 1 \mu \mathrm{g} / \mathrm{ml}$, consistency between testing methods indicates that vancomycin can reliably be reported as susceptible, with MICs $>2 \mu \mathrm{g} / \mathrm{ml}$ reported as intermediate/resistant in line with CLSI/EUCAST guidelines. While vancomycin MICs $\leq 2 \mu \mathrm{g} / \mathrm{ml}$ are susceptible according to both CLSI and EUCAST breakpoints, there is discrepancy between the guidelines regarding resistance, with EUCAST breakpoints stating MICs $>2 \mu \mathrm{g} / \mathrm{ml}$ should be reported as resistant [39], whereas CLSI states that MICs 4-8 $\mu \mathrm{g} / \mathrm{ml}$ 
should be regarded as intermediate and $\geq 16 \mu \mathrm{g} / \mathrm{ml}$ as resistant [37,38]. Of note, vancomycin-intermediate or vancomycin-resistant $S$. aureus strains are rare [20]. The Working Group suggests that if an anomalous MIC is found in the susceptible range (i.e., $>1-\leq 2 \mu \mathrm{g} / \mathrm{ml}$ ), a retest using a different method to determine MIC values should be undertaken. This should be a manual method, either BMD or Etest, then report vancomycin MIC and the method used to test susceptibility, and warn physician of a potential poor outcome. If the value is confirmed, then elevated MIC should be reported, with the treating physician informed of the possibility of poor outcomes in severe illnesses (Figure 1). In addition, the accuracy of vancomycin MIC values obtained by Etest, broth microdilution, Vitek 2, MicroScan or BD Phoenix methods should be checked by regular quality control tests to ensure that values are genuine and are able to guide antibiotic therapy.

\section{Future perspective}

Vancomycin remains the standard of care for most MRSA infections yet methodological difficulties associated with susceptibility testing complicate the precise determination of vancomycin MIC, creating a real challenge for clinicians when selecting the most appropriate antibiotic for the treatment of MRSA infections. It is hoped that improvements in testing methodologies in the future will improve the accuracy (and ideally the timeliness) of MIC determination, enabling clinicians to make informed decisions regarding antibiotic selection with confidence. For institutions with a high prevalence of MRSA, consideration should be given to alternative choices.

\section{Executive summary}

- A working group of infectious disease specialists and clinical/medical microbiologists from Brazil, Canada, Mexico, Saudi Arabia, Russia and the UK has made recommendations for the most suitable methodology to adopt for vancomycin susceptibility testing in order to assist hospitals in monitoring local trends and accurately guide antibiotic therapy for the management of Methicillin-resistant Staphylococcus aureus (MRSA) infections.

Differentiation between community-associated-MRSA (CA-MRSA) \&

hospital-acquired/healthcare-associated-MRSA (HA-MRSA)

- Recent studies suggest a tendency toward a broader mixing among MRSA clones, making appropriate antibiotic selection more challenging.

- While differentiation between CA-MRSA and HA-MRSA strains remains of scientific interest, reporting of the actual antibiotic susceptibility of culture-confirmed MRSA strains is more relevant than information on the origin of the clone.

- Local susceptibility profiles are more critical in the selection of the most appropriate antibiotic since MRSA susceptibility patterns vary across regions.

Reporting susceptibility based on elevated vancomycin minimum inhibitory concentration (MIC)

- Analyses suggest a correlation between vancomycin MIC and outcome. Consequently, accurate measurement of vancomycin MIC is essential in order to ensure correct management of MRSA, reduce inappropriate antibiotic use and lessen the emergence of resistant strains.

- A number of methods are currently used for antibiotic susceptibility testing and can be automated (i.e., Vitek ${ }^{\circledR} 2$, BD Phoenix ${ }^{T M}$, MicroScan and Sensititre ${ }^{T M}$ ) or manual (i.e., disk diffusion, broth microdilution and Etest); however, results are inconsistent accounting for conflicting reports of increases in vancomycin MIC in recent years.

Conclusion \& recommendations

- Microbiology laboratories should report the isolation of MRSA and recount the full susceptibility profile of the organism without any differentiation between CA-MRSA and HA-MRSA.

- Methodological difficulties associated with susceptibility testing create a challenge for clinicians when selecting the most appropriate antibiotic for the treatment of MRSA infections. Until better susceptibility testing methods are available, hospitals must use the methods currently available to them.

- For vancomycin MIC $\leq 1 \mu \mathrm{g} / \mathrm{ml}$, vancomycin can reliably be reported as susceptible. If an anomalous MIC is found (i.e., 1-2 $\mu \mathrm{g} / \mathrm{ml}$ ) retesting using a different method to determine MIC values is advised.

- Susceptibility testing methods should be checked by regular quality control tests to ensure that values are genuine and are able to accurately guide antibiotic therapy. 


\section{Acknowledgments}

The authors acknowledge L Li, Bayer, Berlin, Germany, for her constructive contributions to the discussions of the Working Group. Editorial support was provided by Highfield Communication, Oxford, UK, sponsored by Bayer AG, Berlin, Germany.

\section{Financial \& competing interests disclosure}

The authors received no payment for writing, preparation and approval of this manuscript. Each author received a consultancy honorarium for participation in an advisory board meeting where the issues prompting these recommendations of the Working Group were discussed. Ana Gales received research funding and/or consultation fees from Bayer, Eurofarma, MSD and Pfizer. Sergey Sidorenko received research funding support from Pfizer, MSD and Astellas Pharma Inc., and is a member of the Speaker's Bureau for Pfizer and MSD. The authors have no other relevant affiliations or financial involvement with any organization or entity with a financial interest in or financial conflict with the subject matter or materials discussed in the manuscript apart from those disclosed.

No writing assistance was utilized in the production of this manuscript.

\section{Open access}

This work is licensed under the Attribution-NonCommercial-NoDerivatives 4.0 Unported License. To view a copy of this license, visit http://creativecommons.org/licenses/by-nc-nd/4.0/

\section{References}

Papers of special note have been highlighted as: $\bullet \bullet$ of considerable interest

1. Gould IM, David MZ, Esposito S et al. New insights into meticillin-resistant Staphylococcus aureus (MRSA) pathogenesis, treatment and resistance. Int. J. Antimicrob. Agents 39(2), 96-104 (2012).

2. Shurland S, Zhan M, Bradham DD, Roghmann MC. Comparison of mortality risk associated with bacteremia due to methicillin-resistant and methicillin-susceptible Staphylococcus aureus. Infect. Control Hosp. Epidemiol. 28(3), 273-279 (2007).

3. Bassetti M, Baguneid M, Bouza E, Dryden M, Nathwani D, Wilcox M. European perspective and update on the management of complicated skin and soft tissue infections due to methicillin-resistant Staphylococcus aureus after more than 10 years of experience with linezolid. Clin. Microbiol. Infect. 20(Suppl. 4), 3-18 (2014).

4. Marchaim D, Kaye KS, Fowler VG et al. Case-control study to identify factors associated with mortality among patients with methicillin-resistant Staphylococcus aureus bacteraemia. Clin. Microbiol. Infect. 16(6), 747-752 (2010).

5. David MZ, Daum RS. Community-associated methicillin-resistant Staphylococcus aureus: epidemiology and clinical consequences of an emerging epidemic. Clin. Microbiol. Rev. 23(3), 616-687 (2010).

6. Liu C, Bayer A, Cosgrove SE et al. Clinical practice guidelines by the Infectious Diseases Society of America for the treatment of methicillin-resistant Staphylococcus aureus infections in adults and children. Clin. Infect. Dis. 52(3), e18-e55 (2011).

7. Stevens DL, Bisno AL, Chambers HF et al. Practice guidelines for the diagnosis and management of skin and soft tissue infections: 2014 update by the Infectious Diseases Society of America. Clin. Infect. Dis. 59(2), e10-e52 (2014).

8. David MZ, Dryden M, Gottlieb T, Tattevin P, Gould IM. Recently approved antibacterials for methicillin-resistant Staphylococcus aureus (MRSA) and other Gram-positive pathogens: the shock of the new. Int. J. Antimicrob. Agents 50(3), 303-307 (2017).

9. Sartelli M, Guirao X, Hardcastle TC et al. 2018 WSES/SIS-E consensus conference: recommendations for the management of skin and soft-tissue infections. World J. Emerg. Surg. 13, 58 (2018).

10. van Hal SJ, Lodise TP, Paterson DL. The clinical significance of vancomycin minimum inhibitory concentration in Staphylococcus aureus infections: a systematic review and meta-analysis. Clin. Infect. Dis. 54(6), 755-771 (2012).

-. This meta-analysis reports a threefold increased risk of treatment failure and increased mortality associated with Methicillin-resistant Staphylococcus aureus (MRSA) with an elevated vancomycin minimum inhibitory concentration (MIC), underlining the importance of accurate measurement of vancomycin MIC.

11. Khan A, Wilson B, Gould IM. Current and future treatment options for community-associated MRSA infection. Expert Opin. Pharmacother. 19(5), 457-470 (2018).

12. Bal AM, Coombs GW, Holden MTG et al. Genomic insights into the emergence and spread of international clones of healthcare-, community- and livestock-associated meticillin-resistant Staphylococcus aureus: Blurring of the traditional definitions. J. Antimicrob. Glob. Resist. 6, 95-101 (2016).

-. Reports the emergence of community-associated MRSA (CA-MRSA) clones in healthcare settings, blurring the distinction between CA- and hospital-acquired/healthcare-associated-MRSA (HA-MRSA)

13. Udo EE, Boswihi SS. Antibiotic resistance trends in methicillin-resistant Staphylococcus aureus isolated in Kuwait hospitals: 2011-2015. Med. Princ. Pract. 26(5), 485-490 (2017). 
14. Egea AL, Gagetti P, Lamberghini R et al. New patterns of methicillin-resistant Staphylococcus aureus (MRSA) clones, community-associated MRSA genotypes behave like healthcare-associated MRSA genotypes within hospitals, Argentina. Int. J. Med. Microbiol. 304(8), 1086-1099 (2014).

15. van Hal SJ, Fowler VG Jr. Is it time to replace vancomycin in the treatment of methicillin-resistant Staphylococcus aureus infections? Clin. Infect. Dis. 56(12), 1779-1788 (2013).

16. Sagent Pharmaceuticals ${ }^{\circledR}$. Vancomycin Hydrochloride for Injection, USP (2018). www.sagentpharma.com/wp-content/uploads/2018/09/Vancomycin-PBP_PI_January-2018.pdf

17. Rybak MJ, Lomaestro BM, Rotschafer JC et al. Vancomycin therapeutic guidelines: a summary of consensus recommendations from the Infectious Diseases Society of America, the American Society of Health-System Pharmacists, and the Society of Infectious Diseases Pharmacists. Clin. Infect. Dis. 49(3), 325-327 (2009).

18. Lodise TP, Patel N, Lomaestro BM, Rodvold KA, Drusano GL. Relationship between initial vancomycin concentration-time profile and nephrotoxicity among hospitalized patients. Clin. Infect. Dis. 49(4), 507-514 (2009).

19. Martirosov DM, Bidell MR, Pai MP et al. Relationship between day 1 and day 2 Vancomycin area under the curve values and emergence of heterogeneous Vancomycin-intermediate Staphylococcus aureus (hVISA) by Etest ${ }^{\circledR}$ macromethod among patients with MRSA bloodstream infections: a pilot study. BMC Infect. Dis. 17(1), 534 (2017).

20. Charlton CL, Hindler JA, Turnidge J, Humphries RM. Precision of vancomycin and daptomycin MICs for methicillin-resistant Staphylococcus aureus and effect of subculture and storage. J. Clin. Microbiol. 52(11), 3898-3905 (2014).

21. Wilcox MH, Dmitrieva N, Gales AC et al. Susceptibility testing and reporting of new antibiotics with a focus on tedizolid: an International Working Group report. Future Microbiol. 12(16), 1523-1532 (2017).

22. Hsu DI, Hidayat LK, Quist R et al. Comparison of method-specific vancomycin minimum inhibitory concentration values and their predictability for treatment outcome of meticillin-resistant Staphylococcus aureus (MRSA) infections. Int. J. Antimicrob. Agents 32(5), 378-385 (2008).

23. Swenson JM, Anderson KF, Lonsway DR et al. Accuracy of commercial and reference susceptibility testing methods for detecting vancomycin-intermediate Staphylococcus aureus. J. Clin. Microbiol. 7(7), 2013-2017 (2009).

24. Phillips CJ, Wells NA, Martinello M, Smith S, Woodman RJ, Gordon DL. Optimizing the detection of methicillin-resistant Staphylococcus aureus with elevated vancomycin minimum inhibitory concentrations within the susceptible range. Infect. Drug Resist. 9, 87-92 (2016).

25. Himani AC, Agrawal C, Madan M, Pandey A, Thakuria B. Methicillin resistant Staphylococcus aureus: inconsistencies in vancomycin susceptibility testing methods, limitations and advantages of each method. J. Clin. Diagn. Res. 9(10), DC01-DC04 (2015).

26. Chen SY, Liao CH, Wang JL et al. Method-specific performance of vancomycin MIC susceptibility tests in predicting mortality of patients with methicillin-resistant Staphylococcus aureus bacteraemia. J. Antimicrob. Chemother. 69(1), 211-218 (2014).

27. Eum S, Bergsbaken RL, Harvey CL, Warren JB, Rotschafer JC. Discrepancy in vancomycin AUC/MIC ratio targeted attainment based upon the susceptibility testing in Staphylococcus aureus. Antibiotics (Basel) 5(4), pii: E34 (2016).

28. Silveira AC, Cunha GR, Caierão J, Cordova CM, d'Azevedo PA. MRSA from Santa Catarina State, southern Brazil: intriguing epidemiological differences compared to other Brazilian regions. Braz. J. Infect. Dis. 19(4), 384-389 (2015).

29. Edwards B, Milne K, Lawes T, Cook I, Robb A, Gould IM. Is vancomycin MIC 'creep' method dependent? Analysis of methicillin-resistant Staphylococcus aureus susceptibility trends in blood isolates from north east Scotland from 2006 to 2010. J. Clin. Microbiol. 50(2), 318-325 (2012).

30. Ho PL, Lo PY, Chow KH et al. Vancomycin MIC creep in MRSA isolates from 1997 to 2008 in a healthcare region in Hong Kong. J. Infect. 60(2), 140-145 (2010).

31. Yeh YC, Yeh KM, Lin TY et al. Impact of vancomycin MIC creep on patients with methicillin-resistant Staphylococcus aureus bacteremia. J. Microbiol. Immunol. Infect. 45(3), 214-220 (2012).

32. Pitz AM, Yu F, Hermsen ED, Rupp ME, Fey PD, Olsen KM. Vancomycin susceptibility trends and prevalence of heterogeneous vancomycin-intermediate Staphylococcus aureus in clinical methicillin-resistant S. aureus isolates. J. Clin. Microbiol. 49(1), 269-274 (2011).

33. Chen CP, Liu MF, Lin CF, Lin SP, Shi ZY. The association of molecular typing, vancomycin MIC, and clinical outcome for patients with methicillin-resistant Staphylococcus aureus infections. J. Microbiol. Immunol. Infect. 50(5), 619-626 (2017).

34. Diaz R, Ramalheira E, Afreixo V, Gago B. Evaluation of vancomycin MIC creep in Staphylococcus aureus. J. Glob. Antimicrob. Resist. 10, 281-284 (2017).

35. Gostev V, Kruglov A, Kalinogorskaya O et al. Molecular epidemiology and antibiotic resistance of methicillin-resistant Staphylococcus aureus circulating in the Russian Federation. Infect. Genet. Evol. 53, 189-194 (2017).

36. Tsai S-Y, Chang F-C, Lio C-F et al. Discordance of the vancomycin minimum inhibitory concentration for methicillin-resistant Staphylococcus aureus at $2 \mu \mathrm{g} / \mathrm{mL}$ between Vitek II, E-test, and broth microdilution. Int. J. Antimicrob. Agents 50(S2), S29-S68. OS 1-6 (2017). 
37. Riedel S, Neoh KM, Eisinger SW, Dam LM, Tekle T, Carroll KC. Comparison of commercial antimicrobial susceptibility test methods for testing of Staphylococcus aureus and Enterococci against vancomycin, daptomycin, and linezolid. J. Clin. Microbiol. 52(6), 2216-2222 (2014).

38. CLSI. Performance Standards for Antimicrobial Susceptibility Testing. 28th edition CLSI supplement M100: M100-S22. Clinical and Laboratory Standards Institute, PA, USA (2018).

-• Susceptibility breakpoints for antimicrobial susceptibility testing.

39. European Committee on Antimicrobial Susceptibility Testing. Clinical breakpoints (2018). www.eucast.org/clinical_breakpoints/

-. Susceptibility breakpoints for antimicrobial susceptibility testing. 